\title{
Ab initio Calculation of Quasi-Equilibrium Microcracks in Cubic Boron Nitride
}

\author{
D. GórA* AND K. Parlinski \\ Institute of Nuclear Physics, Radzikowskiego 152, 31-342 Cracow, Poland
}

(Received May 9, 2002; revised version June 21, 2002)

\begin{abstract}
A crack in cubic boron nitride crystal was simulated within the $a b$ initio density-functional theory. The lattice constant, critical stress intensity factor $K_{c}$, which describes crack creation stress, and fracture surface energy $\Gamma$ were calculated from the elongated $2 \times 6 \times 1$ supercell and these quantities agree quite well with the experimental data. We conclude that density-functional theory can be used to estimate crack's material constants.
\end{abstract}

PACS numbers: 71.15.Mb, 75.50.Ee, 62.20.Mk

\section{Introduction}

The renewal of interest to the crack problem was essentially caused by experimental realizations of both equilibrium [1] and dynamic fracture mechanics [2]. Here, we treat the type crack creation, which belongs to the quasi-equilibrium cracks. Our aim is to use the $a b$ initio approach to create the crack, and describe its properties. The quasi-equilibrium cracks are already treated in the classical work of Griffith [3], considered as the beginning of equilibrium fracture mechanics. According to Griffith, to open a crack in a thin plate, the atomic bonds have to be broken into two new free surfaces created inside the plate. For a crack of linear size $l$, the energy cost of this process is of the order of $l$. Simultaneously, the potential energy of the plate under external load is reduced due to the stress relaxation around the crack and this energy decreases as order of $l^{2}$. Thus, the total crack energy reaches the maximum at $l=l_{\mathrm{c}}$. Cracks of length $l<l_{\mathrm{c}}$ tend to disappear, whereas those with $l>l_{c}$ are unstable and will propagate. Basing on these simple

*corresponding author; e-mail: gora@auger.ifj.edu.pl 
ideas and on the elasticity theory the relationship between the critical stress, the crack size, and shape can be derived [5]. Particularly simple is the relation for the straight crack under stress normal to the crack area. In this case the critical stress $\sigma_{\mathrm{cr}}$, and critical crack length $l_{\mathrm{cr}}$ are related as

$$
\sigma_{\mathrm{cr}} \sqrt{\pi l_{\mathrm{cr}}}=K_{\mathrm{cr}}
$$

where $K_{\mathrm{cr}}$ is a material constant called critical stress intensity factor. The $K_{\mathrm{cr}}$ describes the material resistance to formation of a crack.

In this article we undertake, for the first time in our best knowledge, an attempt to create a crack by stress and to calculate its critical stress intensity factor $K_{\text {cr }}$, from the first-principle calculations. As a material we have chosen boron nitride crystal (c-BN) because it has a small number of electrons per atom, and that allows to simulate a relatively large system. The boron nitride occurs in several polymorphic phases, from which at ambient conditions the cubic zinc blende $\mathrm{c}-\mathrm{BN}$ is thermodynamically stable. This material shows extreme hardness and high melting point. It does not dissolve in iron and steel, so it could be used for coating high duty tools.

\section{Calculations}

The ab initio calculations of c-BN structure have been performed using the Vienna ab initio simulation program (VASP) [5, 6], the plane-wave basis, and the fully nonlocal Vanderbilt-type ultrasoft pseudopotentials [7]. The exchange-correlation term, as defined by Perdew and Zunger [8], and the general-gradient approximation of Perdew and Wang [9] were applied. The pseudopotentials for B and $\mathrm{N}$ atoms represent $2 s^{2} p^{1}, 2 s^{2} p^{3}$ electronic configurations, respectively. A plane wave basis set with $400 \mathrm{eV}$ cut-off is used to expand the electronic wave functions. Tests with larger value of cut-off showed very little changes of the total energy. The wave vector summation is confined to the $\Gamma$ point of the Brillouin zone. The ground-state properties of the $\mathrm{c}-\mathrm{BN}$ structure are obtained by minimizing of the total energy with respect to the unit cell volume. For that we have built a $1 \times 1 \times 1$ supercell of c-BN consisting of 8 atoms with periodic boundary conditions and having the symmetry of the space group $F \overline{4} 3 \mathrm{~m}$. The calculated lattice constant $a_{\mathrm{c}}=3.656 \AA$ agree quite well with the experimental value $a_{c}=3.615 \AA$ [10].

The simulation of the crack creation was performed with the $2 \times 6 \times 1$ supercell with 96 atoms and periodic boundary conditions. The supercell was exposed to the uniaxial tension along $\boldsymbol{a}$-axis, so we could define the strain along $a$-axis as $\varepsilon=\left(a-a_{0}\right) / a_{0}$, where $a$ is the current lattice constant of the supercell and $a_{0}=2 a_{\mathrm{c}}$. We expected that a crack will propagate along the longest $\boldsymbol{b}$-axis of the supercell. During the whole simulations, $\boldsymbol{b}$ value was kept constant. However, the $\boldsymbol{c}$ lattice constant was decreased so that the supercell volume $V=568.47 \AA^{3}$ was kept constant. The total energy was minimized with 
respect to changes of $\boldsymbol{a}$-axis and $\boldsymbol{c}$-axis. In each step we increase the $\boldsymbol{a}$ lattice constant by about $0.2 \AA$, decreasing respectively the $c$ lattice constant, and keeping $b$ fixed. In order to create an initial position of the microcrack we displace two boron atoms from the position $(0.5000,0.5833,0.5000) /(0.5000,0.4166,0.5000)$ to $(0.5000-\delta x, 0.5833,0.5000) /(0.5000+\delta x, 0.4166,0.5000)$, where $\delta x=0.005$ is a small displacement along $a$-axis. As a result of the $a b$ initio calculations we have obtained the total energies of $2 \times 6 \times 1$ supercell, the components of the stress tensor, and the relaxed position of $\mathrm{B}$ and $\mathrm{N}$ atoms as a function of strain $\varepsilon$. The results correspond to zero temperature.

\section{Results}

The calculated ground-state energies and $\sigma_{x x}$ component of the stress tensor as a function of strain $\varepsilon$ along $\boldsymbol{a}$-axis are shown in Fig. 1. The ground state energy of the supercell increases with increasing strain. The stress (tension) decreases with strain. For small strains $\varepsilon$ the stress-strain relationship is linear, and hence the elastic properties of c-BN can be elucidated. In cubic symmetry, the $C_{11}$ elastic constant is related with the Hooke law $C_{11}=\sigma_{x x} / \varepsilon_{1}$. From that we find $C_{11}=$ $694 \mathrm{GPa}$. It diverges by $15 \%$ from the experimental value $C_{11}=812 \mathrm{GPa}$ [11].

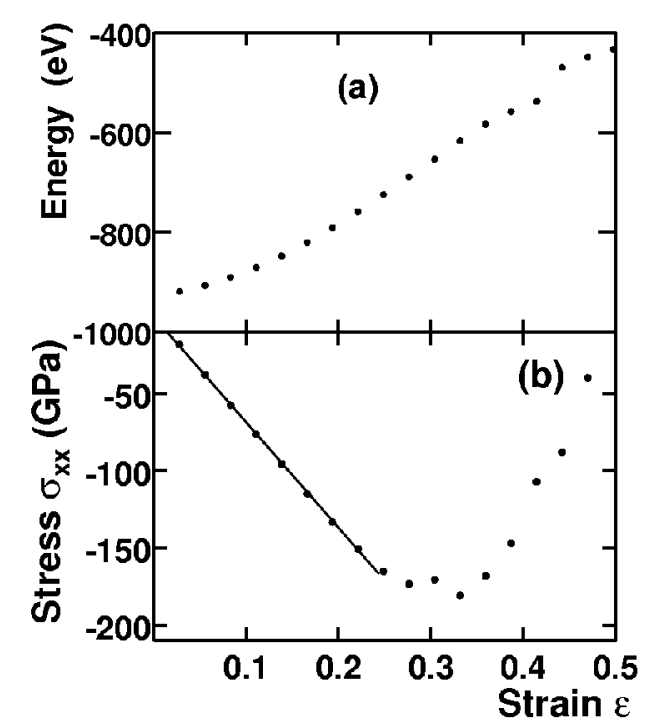

Fig. 1. (a) Ground state energy and (b) $\sigma_{x x}$ component of stress tensor in c-BN as a function of strain $\varepsilon$. Solid line in (b) corresponds to a linear fit in the strain interval $0.0-0.25$.

The changes of the total energy and the $\sigma_{x x}$ component of the stress tensor show unregular behavior around strains $\varepsilon \approx 0.25$ and $\varepsilon \approx 0.41$. In the region of 
small strains $(\varepsilon \leq 0.25)$ the initial crack, i.e. the two slightly displaced atoms, is always annealed out to a perfect cubic structure. At the intermediate strains $(0.25 \leq \varepsilon \leq 0.41)$ the quasi-equilibrium crack along $\boldsymbol{b}$-axis appears as the stable structure. In the highest strain region above $\varepsilon=0.41$ the supercell falls apart, into two separate crystalline pieces. At the critical strain $\varepsilon=0.41$, the critical stress $\sigma_{\mathrm{Cr}}$ along $\boldsymbol{a}$-axis is about $100 \mathrm{GPa}$.

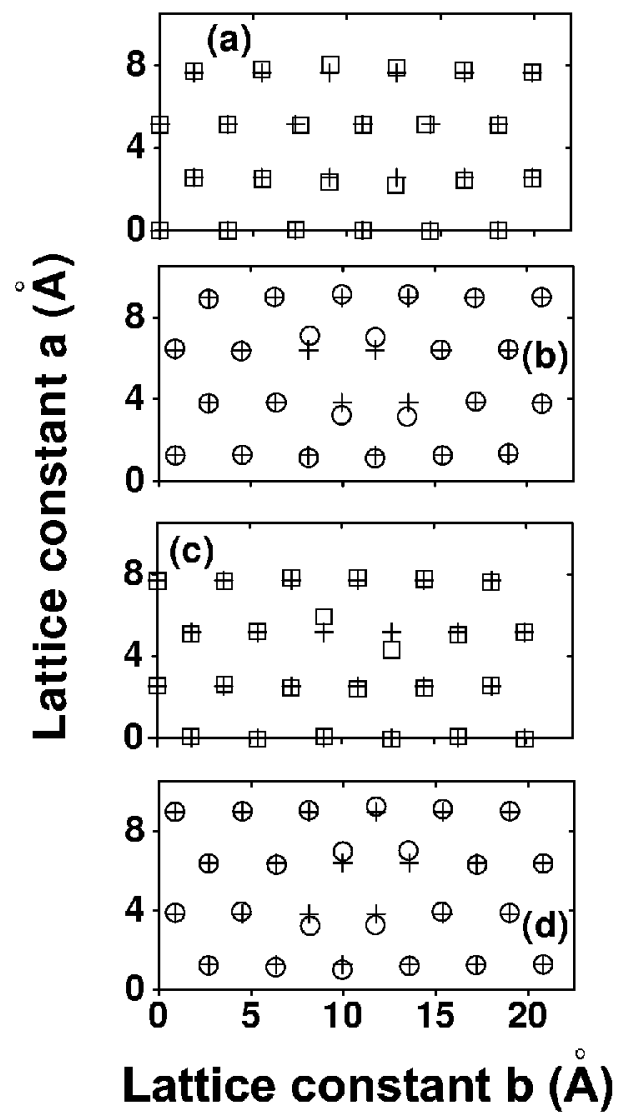

Fig. 2. Simulated atomic positions of boron $(a, c)$ and nitrogen $(b, d)$ atoms in the plane perpendicular to the $\boldsymbol{c}$-axes and for critical strain $\varepsilon=0.41$. A crack is seen in the center of the maps. Squares and circles correspond to relaxed position. Crosses represent perfect lattice. (a,b,c,d) maps correspond to level $z=0, \frac{1}{4}, \frac{2}{4}, \frac{3}{4}$, respectively.

Figure 2 shows the boron and nitrogen positions in the supercell and at the plane perpendicular to the $\boldsymbol{c}$-axis and for the critical strain $\varepsilon=0.41$. Crosses denote the perfect lattice sites. Squares and circles describe the relaxed lattice atomic positions, at the level of $z=0, \frac{1}{4}, \frac{1}{2}, \frac{3}{4}$. Figures $2 \mathrm{~b}$ and $\mathrm{d}$ clearly show that the crack appears in the nitrogen sublattice. In boron sublattice the separation of 
atoms in the region of the crack is certainly smaller. The maximal shift of nitrogen and boron amount $0.78 \AA, 0.62 \AA$, respectively. This distances are sufficiently large to destroy the interatomic attractions.

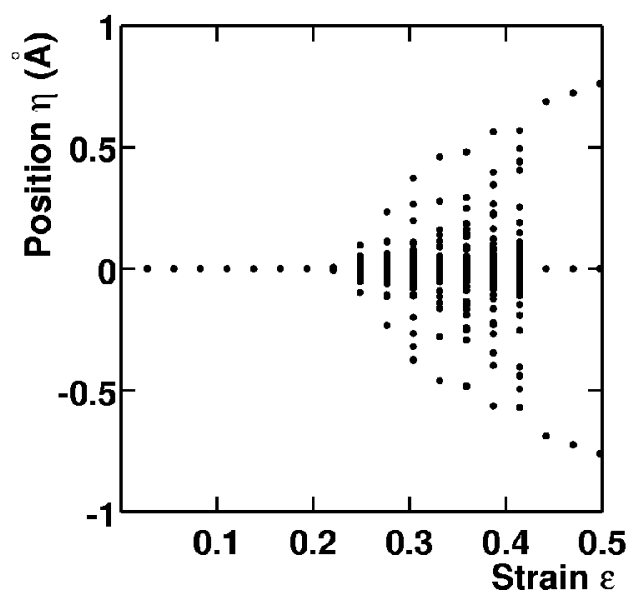

Fig. 3. Reduced $x$-coordinates $\eta$ of all atoms of the supercell as a function of applied strain. In a perfect crystal lattice all $\eta$ should be zero.

Figure 3 presents the reduced $x$-coordinate $\eta=x_{n}-\frac{1}{8} n a$, where $x_{n}$ is the $x$-coordinate of an atom, $a$ is the $\boldsymbol{a}$ lattice constant of supercell at a current stress and $n$ is the index of the atomic plane along $\boldsymbol{a}$-axis. The appearance of crack is manifested by higher scatter of points, which occurs above $\varepsilon=0.25$. Above critical strain $\varepsilon=0.41$ the crystal falls apart.

From Fig. $2 \mathrm{~b}$ and $\mathrm{d}$, one can estimate the length and width of critical crack as $l_{\mathrm{cr}} \approx 7.5 \AA$ and $a_{\mathrm{cr}} \approx 2 \AA$. Knowing that the critical stress is about $\sigma_{\mathrm{cr}}=100 \mathrm{GPa}$ we find from Eq. (1) the critical stress intensity factor as $K_{\mathrm{c}}=4.7 \pm 0.5 \mathrm{MJ} \mathrm{m}^{-5 / 2}$. The material constant $K_{\mathrm{c}}$ is sometimes called fracture toughness. This calculated value agrees very well with the experimental one, which is $5 \mathrm{MJ} \mathrm{m}^{-5 / 2}[12]$.

It is interesting to estimate the values of the surface energy $\gamma$, which measures the amount of energy per unit area needed to form a new fracture surface of the crack. According to the simple theory the crack surface of a solid is unstable for stress higher than the critical one, and

$$
\sigma_{\mathrm{cr}}=\left[2 c_{11} \gamma / \pi l_{\mathrm{cr}}\left(1-\nu^{2}\right)\right]^{1 / 2}
$$

where $\nu=c_{12} /\left(c_{11}+c_{12}\right)$ is the Poisson coefficient. To estimate the surface energy $\gamma$ we have used the following set of parameters: $c_{11} \approx 694 \mathrm{GPa}$; the Poisson coefficient $\nu=0.18$, the critical stress $\sigma_{\mathrm{cr}}=100 \mathrm{GPa}$ and the critical length $l_{\mathrm{cr}} \approx 7.5 \AA$. We find the surface energy to be $\gamma \approx 16 \mathrm{~J} \mathrm{~m}^{-2}$. This value can be compared with the fracture surface energy $2 \gamma$ for borosilicate glass which is around $10 \mathrm{~J} \mathrm{~m}^{-2}$ and with the fracture energy for c-BN which is $2 \gamma \approx 50 \mathrm{~J} \mathrm{~m}^{-2}[13,14]$. It seems that the agreement is reasonable. 


\section{Conclusions}

In summary, we have shown that in the cubic BN the ab initio calculations lead to a correct description of the crack. We have found stable crack by minimization of $2 \times 6 \times 1$ supercell under external uniaxial stress. Our estimated values of elastic constant, critical stress intensity factor and fracture surface energy are in good agreement with experimental data. The present results indicate that the density functional theory can be used to calculate material constants describing characteristic of cracks from first principles.

\section{Acknowledgments}

The authors would like to thank J. Łażewski, and P.T. Jochym for fruitful discussions. This work was partly supported by the State Committee for Scientific Research, grant No. 5 P03B-069-20. The calculations were performed at ACK-Cyfronet, grant SGF2800/IFJ/102/2001.

\section{References}

[1] A. Yuse, M. Sano, Nature (London) 362, 329 (1993); O. Ronsin, F. Heslot, B. Perrin, Phys. Rev. Lett. 75, 2352 (1995).

[2] J. Fineberg, S.P. Gross, M. Marder, H.L. Swinney, Phys. Rev. Lett. 67, 457 (1991); Phys. Rev. B 45, 5146 (1992); J.F. Boudet, S. Ciliberto, V. Steinberg, Europhys. Lett. 30, 337 (1995); E. Sharon, S.P. Gross, J. Fineberg, Phys. Rev. Lett. 74, 5096 (1995).

[3] A.A. Griffith, Philos. Trans. R. Soc. Lond. Ser. A 221, 163 (1921).

[4] B. Cotterel, J.R. Rice, Int. J. Fract. 16, 155 (1980).

[5] G. Kresse, J. Hafner, Phys. Rev. B 47, 558 (1993).

[6] G. Kresse, J. Furhmuller, Software VASP, Vienna, 1999; Phys. Rev. B 54, 11169 (1996); Comp. Mater. Sci. 6, 15 (1996).

[7] D. Vanderbilt, Phys. Rev. B 41, 7892 (1990).

[8] J. Perdew, A. Zunger, Phys. Rev. B 23, 5048 (1981).

[9] J. Perdew, Y. Wang, Phys. Rev. B 45, 13244 (1992).

[10] E. Knittle, R. M. Wentzcovitsch, R. Jeanloz, M.L. Cohen, Nature (London) 337, 349 (1989).

[11] M. Grimsditch, E.S. Zouboulis, A. Polian, J. Appl. Phys. 76, 832 (1994).

[12] T. Taniguchi, M. Akaishi, S. Yamaoka, J. Am. Ceram. Soc. 79, 547 (1996).

[13] K. Reddy, E.H. Fontana, J.D. Helfinstine, J. Am. Ceram. Soc. 71, C310 (1988).

[14] J.A. Hauch, D. Holland, M.P. Marder, H.L. Swinney, Phys. Rev. Lett. 82, 3823 (1999). 